\title{
A Low-Cost Distributed Control Architecture for Intelligent Transportation Systems Deployment in the State of Oklahoma
}

\author{
Robert C. Huck, Joseph P. Havlicek, James J. Sluss, Jr., and Alan R. Stevenson
}

\begin{abstract}
Cost is a major issue for ITS deployment in Oklahoma. We introduce a novel distributed control architecture that has totally eliminated the need for an expensive monolithic traffic management center, thereby dramatically reducing the overall system cost. Analog and digital video streams from the various metro areas are seamlessly integrated by a fault tolerant, dynamically reconfigurable peer-to-peer network of low-cost geographically distributed ITS Consoles operating under the philosophy that any console should be able to control any system resource at any time.
\end{abstract}

\section{INTRODUCTION}

Throughout the USA and in primary urban centers worldwide, traffic on highways and major arterials has increased dramatically in recent decades. Between 1980 and 1999, the number of vehicle miles traveled in US metropolitan areas increased by 76 percent, surpassing the ability of State Departments of Transportation (DOT) to construct roads [1]. According to the Texas Transportation Institute, the cost of congestion delays during calendar year 2000 for the top 75 US metropolitan areas was USD 67.5 billion, which translates to 3.7 billion hours of delay and 5.7 billion gallons of gasoline consumption [2]. Intelligent transportation systems (ITS) have been recognized as an effective means of reducing this growing congestion problem. In the USA, the Federal Intelligent Transportation Systems program was authorized as a result of the Intermodal Surface Transportation Efficiency Act of 1991 and was reauthorized in 1998 by the Transportation Efficiency Act for the 21st Century (TEA21). Via TEA-21, Federal funding is made available to states for the design and deployment of ITS traffic management systems.

The benefits of ITS in terms of improving transportation network efficiency, enhancing safety and security, reducing congestion and travel delay, reducing incident response times, and increasing the efficiency of both transportation and emergency response agencies are well known. A typical ITS comprises vehicle detectors including inductive loops, microwave detectors, and closed circuit television (CCTV)

This work was supported in part by grants from the Oklahoma Department of Transportation and the Oklahoma Highway Safety Office.

R. Huck is with the University of Oklahoma, Telecommunications Interoperability Lab, Tulsa, OK 74135, USA. rchuck@ou . edu

J. Havlicek is with the ITS Laboratory, School of Electrical and Computer Engineering, University of Oklahoma, Norman, OK 73019, USA. joebobaou. edu

J. Sluss is with the ITS Laboratory, School of Electrical and Computer Engineering, University of Oklahoma, Tulsa, OK 74135, USA sluss@ou . edu

A. Stevenson is with the the Oklahoma Department of Transportation, Traffic Engineering Division, Oklahoma City, OK 73105, USA. astevenson@odot.org cameras, fixed and portable dynamic message signs (DMS), highway advisory radio (HAR), an advanced traveler information system (ATIS) which is often Internet based, remote weather stations (RWS), and a typically heterogeneous communications network that links the field hardware to system operators, transportation managers, and emergency management agencies. In most cases, system control is implemented in a centralized traffic management center (TMC) that co-locates the system operators, transportation managers, response agencies, and their dispatchers [3], [4].

A major concern for public planners contemplating the deployment of ITS is the high cost of these systems. For the State of Oklahoma, the cost of constructing and staffing a large, centralized TMC (which is normally expected to be in the regime of USD $\times 10^{8}$ ) was deemed prohibitive. Thus, it became necessary to consider alternative control strategies in order to reduce the overall system cost to a feasible level without compromising system performance and without degrading public perception of the services offered. In this paper, we provide an overview of Oklahoma's statewide ITS architecture and describe a novel distributed control scheme that has totally eliminated the need for an expensive centralized TMC.

The notion of distributed ITS control has been studied previously in a few cases. For example, Dicaf is a completely distributed ITS architecture that addresses vehicle routing by providing dynamic, geographically localized congestion information directly to specialized navigation processors on-board traveling vehicles [5]. These on-board processors utilize the dynamic congestion data to perform optimal route selection in real-time. In the State of Wisconsin, distributed ITS control has been implemented by deploying a network of "local" TMC's that each manage a jurisdictional region and share information throughout the network [6].

The Oklahoma Statewide ITS Console described in this paper is distinct from previous distributed control architectures in that it provides the complete functionality of a centralized monolithic TMC and does not require any specialized invehicle equipment. Any single instance of the ITS Console is capable of functioning independently as a central TMC for the entire State. However, a unique aspect of the Oklahoma architecture is that a large number of these low-cost consoles are deployed to operate simultaneously in a fault tolerant peer-to-peer network. This results in a virtual TMC where the various system operators, transportation managers, and incident management agencies can remain geographically distributed in their current facilities throughout the State but still enjoy most if not all of the benefits provided by a large, 
centralized TMC environment.

\section{REQUIREMENTS \& ARCHITECTURAL CONCEPT}

Oklahoma's statewide ITS currently covers two geographically separated major metropolitan areas, viz. Oklahoma City and Tulsa. ODOT also has a 20-year plan that will incorporate most of the rural highways and smaller metropolitan areas into the Statewide ITS infrastructure. As a minimum set of functional requirements, the ITS must provide:

- Incident Management - incident detection information relayed to traffic managers for verification, assessment, and timely dispatch of appropriate response teams.

- Work Zone Traffic Management - traffic volume, speed, and queue information relayed to traffic managers.

- Weather Information Monitoring - weather and pavement sensor information provided to traffic managers and road maintenance crews.

- Critical Infrastructure Monitoring - monitoring of airports, water ports, and major highway interchanges for detection of incidents or shutdowns.

- Commercial Vehicle Operations - afford ease of travel through the State by allowing electronically tagged vehicles to process credentials in motion and to be weighed in motion.

- Public Dissemination of Information - messages and alerts posted to dynamic message signs, a web-based ATIS, and a 511 traveler information system; near realtime still images posted to the ATIS.

To meet these functional requirements, a wide array of field equipment is being deployed, including dedicated fiber optic cables, communication network hardware, pan-tiltzoom (PTZ) CCTV cameras to provide full-motion video for incident management, web cameras to provide low rate video and still images for public dissemination, DMSs, Remote Traffic Detectors (RTD), and RWSs.

Design requirements for the ITS Console are driven by a philosophy which insists that a sufficiently privileged user should be able to log into an ITS Console anywhere in the State and

1) control any ITS device at any time,

2) see video or images from any camera at any time,

3) post warnings, alerts, or informatory messages to all ITS Consoles statewide at any time,

4) post warnings, alerts, informatory messages, or images to the public ATIS and 511 system at any time, and/or

5) provide video from any CCTV camera to designated public and private agencies at any time.

This philosophy is consistent with the National Cooperative Highway Research Program findings that "Interagency exchange of information promotes rapid, efficient, and appropriate response from all agencies" [7]. In addition, due to the critical nature of the system, it must be fault tolerant; localized failures should not preclude the system from performing the rest of its non-failed functions. If an ITS Console or an ITS device has failed, the rest of the operators and devices must remain functional.
Through its user interface, the ITS Console must provide a geo-referenced graphical representation of the State that shows major and secondary roadways and is both panable and zoomable. It must support immediate jumping to predefined views including, e.g., the Oklahoma City and Tulsa metroplexes. Where available, aerial photography will be overlayed on the map display when the appropriate zoom level is attained. ITS devices must be depicted by graphical icons that indicate location, status, and type of equipment and, when clicked, provide full control of the equipment. Graphical icons depicting incidents and work zones must also be easily added, deleted, edited, and exported to the public ATIS and 511 system.

Some of the agencies that are or will be connected to the statewide private network of ITS Consoles include ODOT Traffic Engineering and Maintenance Divisions, ODOT Division Engineers, Civil Emergency Management and Homeland Security, 911 dispatchers, Emergency Management Services, local and state police agencies, fire departments, the National Guard, and the Governor's Office. Each authorized user at each agency is provided with an ITS Console that is connected through the private network to all other ITS Consoles statewide in a peer-to-peer network. A multi-tiered system of user levels and user privileges is implemented to manage access to system resources and mediate critical sections. The ITS Console provides an "instant messaging"like capability whereby one user can gracefully request access to a system resource that is currently under the control of another user, although a higher privileged user can always preempt a resource from a lower privileged user when necessary. Any given user maintains their level and privilege structure when logging in to an ITS Console anywhere in the State.

Nominally, a full-function ITS Console provides video distribution functions and control of CCTV video cameras and Internet protocol (IP) web cameras, identification, reading, and posting of incident and work zone locations and information, and control of DMSs for directly disseminating a variety of information to drivers including the current state of incidents, congestion, and detours, critical weather alerts, evacuation routing, and AMBER (America's Missing: Broadcast Emergency Response) alerts. Sharing of CCTV camera video signals between agencies is carried out as agreed upon in specific interagency memoranda of understanding. In addition to the full-function ITS Consoles, there are also limited capability "read only" consoles available to certain private entities such as news media that have a need for access to traffic related information.

General public access to the information provided by the ITS is through a web-based ATIS and a planned 511 traveler information system, both of which will be automatically populated with data from the network of ITS Consoles. One unique aspect of the Oklahoma system is that the CCTV camera video streams are reserved for incident management and will not be available to the general public. However, a set of four IP web cameras will be co-located with each CCTV camera to provide near real-time still images for 
public consumption. Fully privileged ITS Console users have the capability to block the still images from individual web cameras in cases where public safety or security are at issue. An independent network of microwave RTDs is being deployed by Mobility Technologies, Inc. (www.traffic.com), and will provide traffic speed data for color coding roadway segments on the ITS Consoles and ATIS.

\section{SYSTEM IMPLEMENTATION}

\section{A. Communications Network}

Through public-private and public-public partnership agreements, ODOT has acquired a large inventory of fiber optic cable that runs throughout the State. These agreements typically allow telecommunications companies onto controlled access right-of-ways in exchange for a specified number of fiber strands and some number of empty conduits to be placed in the ground in conjunction with their build. These fiber optic cables and conduits become the property of the State for exclusive use by ODOT.

A dedicated Gigabit Ethernet (GigE) network was created in and around Oklahoma City for the ITS network backbone. Dedicated fiber optic cables were not available in Tulsa, so the ITS backbone in that metropolitan area was constructed using Virtual Local Area Network (VLAN) connections on a shared GigE network in cooperation with the Oklahoma Transportation Authority (OTA), which is the agency responsible for operating the State's turnpike system. In order to connect Oklahoma City and Tulsa, there was again no dedicated fiber optic cable available and a VLAN connection was made on the OTA GigE network. In the 20-year ITS deployment plan, additional fiber optic cables will eventually be installed to allow for a dedicated ITS network backbone in Tulsa and for the interconnection between Oklahoma City and Tulsa. ITS devices are not connected directly to the GigE network since this would constitute an inefficient use of the GigE ports. Instead, 100 Megabit spurs are deployed in daisy chains to transport data from one equipment location to another until the aggregate data rate reaches a level that is practical for connection to the GigE backbone. These aggregated GigE connections generally occur at communication huts where GigE switches are installed.

Fault tolerant rings are installed where fiber is available and are also planned for future construction projects at locations where fiber is not currently available. There are a number of off-the-shelf switches currently being manufactured that meet the operational temperature range requirements for installation in standard roadside communications cabinets. Optical switches, however, still require a controlled operating environment and are therefore installed in communications huts equipped with air conditioning. The capacity of the GigE network will allow all operators and ITS device traffic to move around the network and meet the system requirements as articulated in [8]. For this system, two-plus-two spare fibers are required for both the GigE network and the 100 Megabit network.

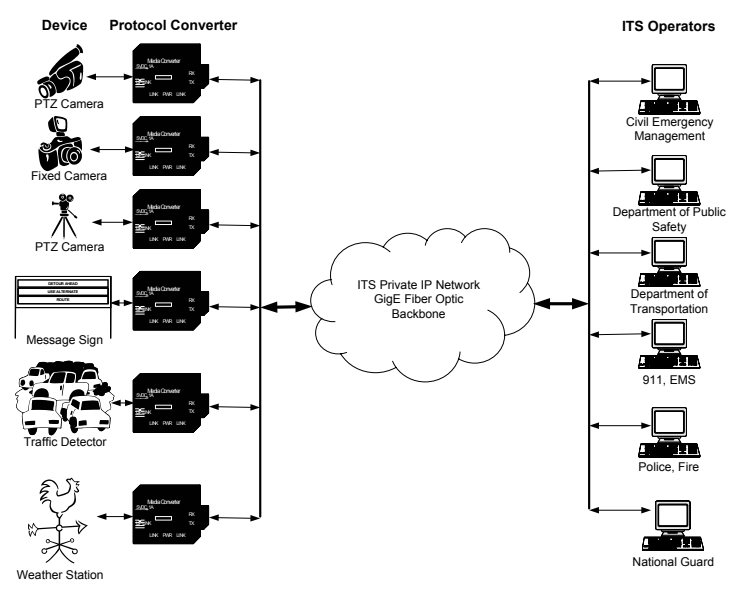

Fig. 1. Distributed IP network architecture.

In areas where fiber optic cable is not currently available, wireless network links are installed as a temporary solution. For these connections, the Motorola Canopy ${ }^{\mathrm{TM}}$ system was chosen and has thus far proven reliable for this application. Access points were installed on camera poles where fiber optic cables were available and subscriber modules were installed at the remote devices. The Canopy ${ }^{\mathrm{TM}}$ system provides 6.2 Mbps of bandwidth point-to-multipoint with a range of two miles [9]. By installing a passive reflector, the range is increased to greater than 10 miles, but communications are limited to point-to-point only [10]. A single Canopy ${ }^{\mathrm{TM}}$ link provides device control simultaneously with acceptable video quality for either a single CCTV camera video stream or multiple IP web camera video streams.

The architecture of the distributed IP network is shown in Fig. 1. In addition to video streams, communication with ITS devices including control and data acquisition for RTDs and RWSs is also performed over this network. Either a single port serial server or a terminal server is used to attach an EIA-232 or EIA-422 device to the IP network. The terminal server devices are IP addressable, off-the-shelf, and handle the protocol conversion from EIA-232 or EIA-422 to IP seamlessly. The posting of DMS messages using the IP network is much faster than the previous implementation consisting of dial-up modems. Moreover, through the use of sockets, multiple DMSs can be controlled simultaneously. This simultaneous control enables AMBER alerting to occur much faster than before since messages can be posted to all selected signs throughout the State at the same time.

\section{B. Analog Video Feeds}

In the field, the CCTV cameras and IP web cameras are installed on 63 foot (19.2 meter) and 84 foot (25.6 meter) steel poles, on DMS sign structures, and, in locations where a new pole installation is impractical, on traffic signal poles. For the CCTV cameras, analog as opposed to digital video is deemed preferable when practical. Although multiple analog video streams can be multiplexed onto a single fiber, each individual camera requires a dedicated fiber for transmission 


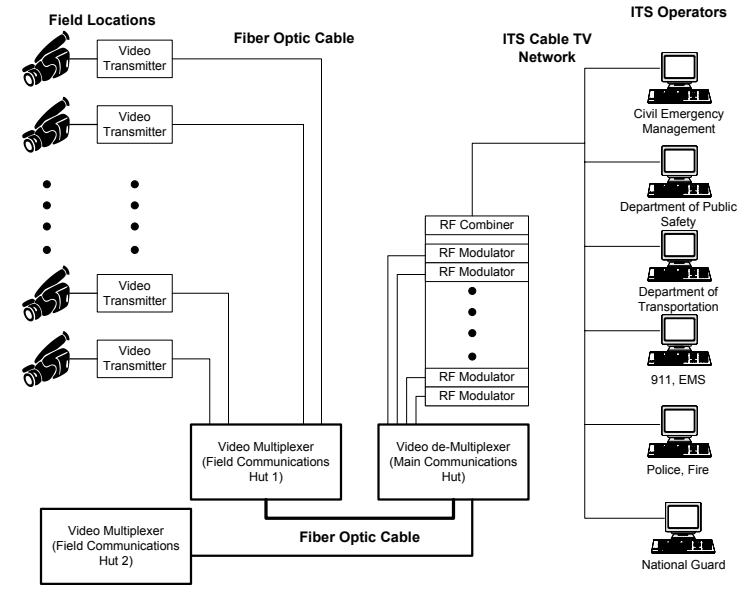

Fig. 2. Analog video distribution scheme for the Oklahoma City metro area. Individual video streams are multiplexed together and transmitted to the central communications hut where they are demultiplexed and used to generate a private CATV signal that is broadcast to the ITS Consoles.

of the video stream from the pole to the multiplexer location. Thus, implementation of analog video feeds is practical only in areas where the available fiber is plentiful. For the statewide ITS, this implies that analog video is feasible only in the Oklahoma City metro area. Throughout this area, a fiber optic video modem is installed with each camera and a dedicated fiber is used to transmit the video stream to a communications hut where multiple discrete video signals are multiplexed together on a single fiber. The multiplexed analog video signals are all transmitted to a central communications hut where demultiplexers are used to recover the individual discrete video streams.

At the central communications hut, RF modulators and combiners are used to generate a private cable TV (CATV) signal such that the analog video streams from individual cameras can be accessed with a standard TV tuner. As depicted in Fig. 2, this CATV signal is broadcast over a dedicated fiber to all agencies connected to the ITS private network in the Oklahoma City metro area. In this configuration, three fibers are required to connect an agency to the ITS private network for control and viewing of ITS assets: two fibers for the 100 Megabit Ethernet connection and a third fiber to receive the private CATV broadcast.

\section{Digital Video Feeds}

A digital video scheme was implemented for the CCTV cameras installed in locations such as Tulsa where fiber assets are limited. This approach generally requires installation of a digital video encoder with each camera. The encoder output can be connected directly to the 100 Megabit or GigE networks. Although most modern codecs provide multiple video inputs from among which one may be selected at any time, sharing a single encoder between multiple cameras is undesirable in this application first because it requires dedicated fiber connections between each camera and the corresponding encoder input and second because such a

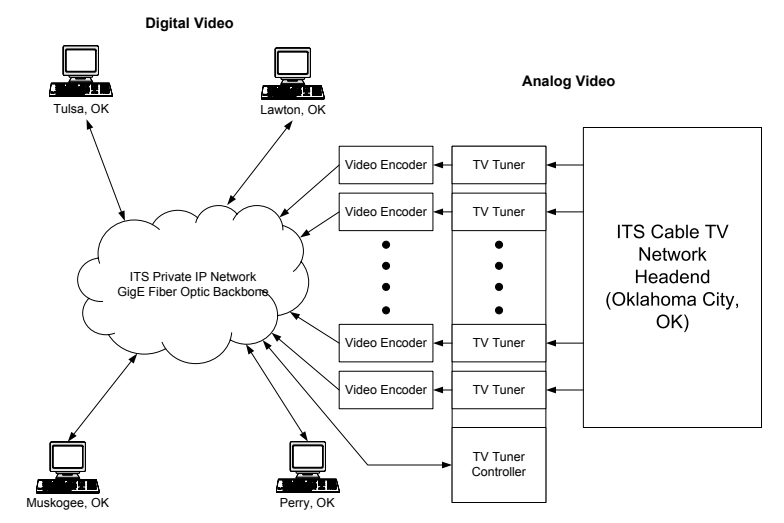

Fig. 3. Hardware mechanism for providing statewide access to analog video signals originating in the Oklahoma City area. In response to a service request, the desired video stream is selected from the CATV system and routed onto the GigE network through a digital video encoder.

configuration precludes the possibility of one or more users being able to simultaneously view the two video streams from two cameras connected to the same encoder.

MPEG-2, MPEG-4, and MJPEG codecs from several manufacturers were fielded and tested. MPEG-2 was ultimately selected as the best tradeoff between compression performance and video quality. For agencies where several ITS Consoles are located in close proximity, it may be feasible to share a common hardware decoder between them and display the decoded video on a peripheral monitor. However, current plans call for software decoding to be present on all instances of the ITS Console and to be utilized as the primary decoder in a majority of cases. Low-cost hardware assisted solutions will be considered in the event that throughput issues arise with the software decoder.

\section{Mixed-Mode Video Sharing and Interoperability}

A digital video stream originating in, e.g., Tulsa can be routed to any ITS Console in the State via that console's 100 Megabit network connection. Fig. 3 illustrates the somewhat more complicated means by which an analog video signal originating in the Oklahoma City metro area can be viewed on ITS Consoles located in Tulsa, Lawton, and other areas that are connected to the ITS network backbone but do not have direct access to the private CATV broadcast signal. The central communications hut in Oklahoma City is equipped with a video switch, a bank of TV tuners, and a bank of video encoders. When an ITS Console located in, e.g., Lawton requests an analog video stream from the CATV system, this stream is selected by one of the tuners and routed through an encoder onto the GigE network. An increasing number of tuners and encoders will potentially be required to meet the demand as the number of ITS Consoles with IP-only connections to the network increases throughout the 20-year statewide deployment plan. 


\section{E. IP Web Cameras}

Multiple IP web cameras each providing a fixed view are typically installed with every CCTV camera. Since use of the CCTV cameras is reserved for incident management, the IP web cameras are an important source of images for automatically populating the public ATIS. With this approach, an ITS Console user can utilize a CCTV camera for assessing and managing an incident without disrupting the public distribution of traffic images via the ATIS. Each IP web camera is equipped with a small Linux server onboard that provides an inherently digital IP network interface. At one minute intervals, a still image is acquired from each IP web camera and sent to the ATIS using File Transfer Protocol (FTP). Each new image overwrites the preceding one from the same location, as there is currently no provision for archiving images or video anywhere in the statewide ITS architecture.

\section{ITS CONSOLE}

Each instance of the statewide ITS Console is an offthe-shelf, Intel-based PC type platform running under Microsoft Windows XP. A concerted effort has been made to base the software architecture on open source and public domain packages where possible in order to avoid costly and recurrent software licensing fees. The following packages are required to support the ITS Console main application software:

- Apache web server (the Apache Software Foundation, www.apache.org). The ITS Console main program is a web-based application that runs in a specially configured instance of the Microsoft Internet Explorer. However, at no time is it envisioned that any ITS Console will have access to the World Wide Web (WWW); rather, the application consists of a collection of pages that are sourced from the local Apache server resident on-board each individual console.

- PHP scripting language (the PHP Group, www.php.net). PHP is a hypertext preprocessor that facilitates webbased software development.

- MySQL (MySQL AB, www.mysql.com). MySQL is a popular, open source database server product.

- MapServer GIS (Regents of the University of Minnesota, mapserver.gis.umn.edu). MapServer is a CGI application that facilitates and supports the development of web-based geographical information systems (GIS).

Within this environment, the main application software was developed using a heterogeneous mix of Microsoft Visual Basic, Visual C++, JavaScript, and PHP. This software was developed such that each ITS Console is capable of functioning as a stand alone TMC console, of displaying analog and digital video, of controlling the CCTV cameras and DMSs, and of controlling, configuring, and acquiring data from RTDs, RWSs, and all of the other various ITS sensors and detectors that have been deployed. The control system is completely distributed in the sense that, on a dynamic basis, any ITS Console is capable of controlling any device that it can communicate with.
It is also fault tolerant in the sense that any group of one or more ITS Consoles, when connected together through a network or a subnet thereof, will cooperate to provide control of all ITS devices connected to the net on an instantaneous basis. This is accomplished by implementing a sophisticated message passing queue between all ITS Console instances that are mutually visible to one another. The network of ITS Consoles is peer-to-peer but not Ad Hoc. Each console runs a common software program and maintains a common database that includes complete information about all authorized ITS Console users and about all ITS Console instances. Database synchronization is maintained via the queue. In cases where a particular console is required to send messages to a second console that is currently offline or not visible, those message are queued until the destination console once again becomes visible.

When a given console desires to take control of a certain device, the console operator selects the device via a mouse click and a message is sent through the queue to all ITS consoles. If another user is already controlling the desired device, the operator is alerted by displaying the controlling user name, organization, and phone number. The requestor can contact the controller using a built in "instant messaging"like facility and ask that the device be released. This provides for a graceful transfer of semaphores. In case the controller is nonresponsive, a user with a higher level can always preempt the device. This is useful, for example, if an incident occurs and a Department of Public Safety (DPS) officer wishes to view the incident but the controller is unavailable to release the camera. In such cases, control is released by a message through the queue and is then granted to the requestor by the software. All such transactions are performed with the use of messages transmitted through the queue.

A number of other benefits are realized through implementation of the queue structure. Command logs are maintained for records keeping and troubleshooting. All actions performed by all operators are maintained in a command $\log$ that is stored in the common database. Error messages are also logged in the database and are accessible remotely by system maintenance personnel. The queue system allows orderly control of all ITS devices. All operators that are able to communicate with a device are also synchronized with other operators in contact with that device. This queuing system keeps the multiple ITS Console databases updated and maintains synchronization between them. In case an ITS Console is isolated due to, e.g., a backhoe cutting its connecting fibers, the queue for that console is immediately updated when the console is reconnected with the larger network. A system of time stamps prevents stale messages from corrupting the database of any given console when it is reconnected to the network.

User levels and privileges are defined to represent the type of agencies and their functions that use the system. Currently there are ten levels and 17 privileges. Each operator can be assigned to any configuration of level and privileges and this information is stored in the common database. When administrators make changes to the level or privileges of a 
user, messages are sent through the queue to update all other console databases. Through this process, any ITS operator can log in to any ITS console and maintain their assigned capability to operate the ITS system.

It should be noted that external monitors, including wall mounted plasma displays, can be controlled by the ITS console through external TV tuners connected through terminal servers. This capability provides for on-the-fly configuration of a centralized Traffic Management Center (TMC) or Emergency Operations Center (EOC) at any given location when the need arises. Additionally, with this implementation, a remote TMC can be established using wireless links if fiber is not available at the desired location.

\section{FIBER ISSUES}

In this section we discuss a few important issues concerning the fiber optic infrastructure, which is perhaps the most important single element of the ITS. Given the system reliance on high volume network traffic for encoded video, control of ITS devices, and data acquisition, fiber optic cables provide the best telecommunications medium currently available. The cost of fiber optic cable installation is high and currently prohibits widespread deployment of ITS in many rural areas of the State. Based on recent projects put out for competitive bid in Oklahoma, fiber optic cable installation currently costs approximately USD $20 \mathrm{~K}$ per mile in rural areas and about USD $60 \mathrm{~K}$ in urban areas. The main reason for the cost difference between rural and urban installations is the need for boring as opposed to trenching in the latter. Wireless technologies provide many alternative communication modalities, but none of them are currently equivalent to fiber.

Each analog video signal requires a dedicated fiber optic cable from the camera pole to the point where the signal can be multiplexed in a cost effective manner. Digital video codecs provide an alternative where a high bandwidth packet switched IP network can be run all the way to the camera pole. However, the digital video delivered by currently available, real-time field-worthy encoders is deemed "adequate" but not desirable. In particular, the digital video streams have an "unnatural" look and invariably involve a coding delay that is problematic when panning, tilting, and zooming a camera for incident management. The private CATV system implemented in the Oklahoma statewide ITS is a novel and cost effective means of broadcasting a large number of highquality analog video signals to geographically distributed ITS Consoles. However, it can only be received in areas where three dedicated fibers are available for implementing the ITS private network, which excludes the Tulsa metro as well as many rural areas. As shown in Fig. 3, the solution we have implemented allows consoles in these areas to view the video streams on the private CATV system by switching individual analog video streams through a bank of digital encoders and onto the GigE backbone. This solution is practical and scalable to a point, but the size and cost of the digital encoder bank will potentially grow linearly with the number of ITS Consoles having IP-only network connectivity as ITS deployment expands into the rural areas of the State.

Currently, accidental fiber optic cable cuts constitute the most serious threat to the statewide ITS. Without physically separated redundant network paths, the system is susceptible to network failures. When a cut does occur, the unique distributed control architecture implemented in the Oklahoma system enables any group of connected ITS Consoles to continue functioning together and to provide complete control of all the ITS devices that are still visible. Obviously, however, the devices that are no longer visible cannot be controlled. As network connectivity is restored, the ITS Consoles automatically and dynamically resynchronize with one another.

\section{CONCLUSION}

We briefly described the main features of the statewide ITS that is currently under deployment in Oklahoma and introduced a novel distributed control architecture that has totally eliminated the need for an expensive, centralized traffic management center (TMC). The system seamlessly integrates both analog and digital video streams under a philosophy which insists that all devices be accessible from all ITS Consoles statewide at all times. A potentially large number of geographically distributed ITS Consoles are connected in a fault tolerant, dynamically reconfigurable peer-to-peer private network. This approach effectively realizes a "virtual TMC" that enables the involved agencies to remain geographically distributed in their current facilities and thereby avoids the substantial cost of a single monolithic statewide TMC. As the need arises, the system also supports dynamic consolidation of personnel and resources to configure a centralized TMC or EOC on-the-fly in response to critical events.

\section{REFERENCES}

[1] R.P. Maccubbin, B.L. Staples, and M.R. Mercer, Intelligent Transportation Systems Benefits and Costs: 2003 Update, Mitretek Systems, Inc., Washington, DC, May 2003, pp. 1-14.

[2] D. Schrank and T. Lomax, "The 2002 Urban Mobility Report", Texas Transportation Institute, The Texas A\&M University System, College Station, TX, June 2002, pp. 20-23.

[3] R.H. Brakman and J.J. Limarzi, "ITS at the Hudson Valley transportation management center," IEEE Intell. Syst., vol. 19, no. 3, May-June 2004, pp. 8-12.

[4] M.J. Kelly, D.J. Folds, N. Sobhi, "ATMS 2000: Hybrid automation or a lights out traffic management center?," in Proc. Nat. Telesyst. Conf., June 16-17, 1993, pp. 37-42.

[5] N. Utamaphethai and S. Ghosh, "Dicaf: a distributed architecture for intelligent transportation," IEEE Computer, v. 31, no. 3, March 1998, pp. 78-84.

[6] J. Wu, L. Henson, and J. Amidon, "Distributed concept in ATMS software," in Proc. IEEE Intell. Transp. Syst. Conf., Oct. 12-15, 2003, pp. 1295-1298.

[7] K. Brooke, K. Dopart, T. Smith, and A. Flannery, Sharing Information between Public Safety and Transportation Agencies for Traffic Incident Management, Mitretek Systems, Inc., Washington, DC, 2004.

[8] "Oklahoma Statewide ITS Strategic Plan", Oklahoma Department of Transportation, Oklahoma City, OK, February 2003.

[9] Motorola Canopy ${ }^{\mathrm{TM}}$ Access Point Specification. Available: http://motorola.canopywireless.com/prod_specs.php?action=show\&recID=1392 (visited February, 2005).

[10] Motorola Canopy ${ }^{\mathrm{TM}}$ Subscriber Module Specification. Available: http://motorola.canopywireless.com/prod_specs.php?action=show\&recID=1394 (visited February, 2005). 